\title{
The Discrimination of Structure: III. Representation of Spatial Relationships
}

\author{
Mark Haselgrove, David N. George, and John M. Pearce \\ Cardiff University
}

\begin{abstract}
Pigeons received a discrimination in which the spatial relationship between 2 adjacent rectangles filled with different colors signaled the trial outcome. Test trials then involved the same rectangles separated horizontally by a gap. The tests in Experiment 1 disrupted the discrimination more when the rectangles were tall and thin than when they were short and wide. Experiment 2 revealed that the width of the rectangles rather than their height determined the extent to which separating them would disrupt the original discrimination. The results are explained in terms of a template-matching account of pattern recognition with the additional assumption, supported by Experiment 3, that the size of a template can be altered to improve its match with a test pattern.
\end{abstract}

Keywords: discrimination, structure, template

The present experiments were conducted to elucidate the way in which animals represent the spatial relationship between the components of a visual pattern. An autoshaping experiment by George, Ward-Robinson, and Pearce (2001) demonstrates that pigeons are sensitive to this type of information. Subjects were shown six patterns, each of which was composed of two adjacent colored circles. Patterns in which the colors of the circles were red to the left of green (R-G), green to the left of blue (G-B), and blue to the left of red (B-R) signaled one outcome, whereas the reflections of each of these patterns, G-R, B-G, and R-B, signaled the opposite outcome (see Table 1). Pigeons were able to solve this discrimination, which indicates their behavior was under the control of the spatial relationship between the colors of the two circles. If a simpler design had been used, based on a discrimination between just one pattern and its reflection, then it would not be possible to draw this conclusion. Instead, the birds may have adopted the strategy of looking consistently at one side of the pattern and responded on the basis of its color.

George et al. (2001) referred to the task just described as a spatial structural discrimination because its solution relies on an appreciation of how the components of each pattern are spatially related, or structured. Theories of pattern and object recognition provide at least two different accounts for the way in which such spatial information might be encoded. According to one class of theory, patterns and objects are represented by structural descriptions, which contain information about the spatial relations between their parts (e.g., Hummel \& Biederman, 1992). Thus, a

Mark Haselgrove, David N. George, and John M. Pearce, School of Psychology, Cardiff University, Cardiff, United Kingdom.

This work was supported by Biotechnology and Biological Science Research Council of the United Kingdom Grant 72/S17198. We are grateful to Tim Pearce for his help with the experiments.

Correspondence concerning this article should be addressed to John M. Pearce, School of Psychology, Cardiff University, Tower Building, Park Place, P.O. Box 901, Cardiff CF10 3YG, United Kingdom. E-mail: pearcejm@cardiff.ac.uk training pattern in the experiment by George et al. (2001) might be represented by the structural description "red to the left of blue." Each of the six training patterns would be represented by a different structural description. If these descriptions were associated with the outcome that followed the pattern, it would then be possible to identify the patterns that did, or did not, signal food. Although this class of explanation was originally developed with perception by humans in mind, it has been applied to studies of discrimination learning in animals (Kirkpatrick-Steger \& Wasserman, 1996; Van Hamme, Wasserman, \& Biederman, 1992; Wasserman et al., 1996; Wasserman, Kirkpatrick-Steger, Van Hamme, \& Biederman, 1993).

A rather different account for the solution of the discrimination can be based on theories that assume that patterns are represented by templates. These accounts have been applied to findings with both humans (e.g., Tarr \& Bülthoff, 1998; Ullman, 1998; see Palmer, 1999, for a review) and animals (Blough, 1985; Cartwright \& Collett, 1983; Gochin, 1996; Heinemann \& Chase, 1990; Srinivasan, 1992; Wasserman et al., 1996; Wehner, 1997). According to this type of account, exposure to a pattern will result in the formation of a template that has been likened to a "mental snapshot" (Cartwright \& Collett, 1983). A subsequent presentation of the pattern is then assumed to result in it being compared with the template to determine whether the perceived pattern is the same as the remembered one. Pigeons in the study by George et al. (2001) would, therefore, be assumed to acquire templates based on the six training patterns, and each template would be associated with the relevant outcome. Of course, variations in the distance and angle from which a pattern is viewed may result in there being more than one template for each training pattern. These variations allow the templates to be used more flexibly than if only a single template is formed of a training pattern (Blough, 1985; Heinemann \& Chase, 1990; Tarr \& Bülthoff, 1998). By identifying the template to which it corresponds, the currently perceived pattern can then be classified correctly. If the correspondence is not perfect, then it should still be possible for the pattern to elicit a response. Theories of template matching in animals (e.g., Heinemann \& Chase, 1990) 
Table 1

Combinations of Colors That Filled the Adjacent Circles in Experiments by George et al. (2001) and Adjacent Rectangles in Experiments 1-3

\begin{tabular}{ll}
\hline One outcome & Opposite outcome \\
\hline Red-green & Green-red \\
Green-blue & Blue-green \\
Blue-red & Red-blue \\
\hline
\end{tabular}

Note. The left- and right-hand colors of each pair indicate, respectively, the colors of the left- and right-hand circles or rectangles.

generally assume that the degree to which a test pattern matches a template for a training pattern determines the extent to which the response to the training pattern will generalize to the test pattern.

The overall concern of the present experiments is to attempt to determine whether the acquisition of structural descriptions or templates underlies the ability of pigeons to solve a spatial structural discrimination. The experiments will have important implications for our understanding of the representational ability of this species. If it can be shown that pigeons rely on structural descriptions to solve the discrimination, then it would suggest they can use propositions of the form "red to the left of blue," which, in turn, would imply a capacity for acquiring symbolic or abstract representations. Alternatively, if the results suggest that the formation of templates is responsible for the solution of the discrimination, then it would imply a less sophisticated representational ability. All that would be acquired is a concrete copy of the training patterns. According to this account, the ability to solve a spatial structural discrimination depends on mechanisms that may be no more sophisticated than those involved in a simple discrimination between, for example, a red circle and a green circle.

\section{Experiment 1}

In keeping with the study by George et al. (2001), two groups of pigeons were trained to solve a spatial structural discrimination using three colors and the six configurations listed in Table 1. Instead of being used to fill adjacent circles, the colors were presented in adjacent rectangles. The rectangles shown in the upper row of Figure 1 formed the basis of the training patterns for the vertical and the horizontal groups in the experiment. The patterns were presented on a TV monitor, which was dark during the intertrial interval and in the region not occupied by the patterns during a trial. The method of conditioning was autoshaping, and as training progressed, it was expected that responding on a key in front of the TV screen would become more rapid during the three reinforced patterns than during the three nonreinforced patterns.

Once the discriminations had been mastered, test trials were given in which the rectangles of a pattern were separated by a gap that was equal in length to the longer side of the rectangles (see middle row of Figure 1). If the solution of the structural discrimination depends on the acquisition of a template of each training pattern, then the groups should respond differently on the test trials. Suppose that the comparison between the remembered template and the perceived patterns involves sliding one over the other until the largest degree of overlap is found (Blough, 1985). Given the distance between the rectangles of the test patterns for the vertical group, the closest match between one of these patterns and the template of the training pattern on which it was based will occur when a single rectangle from a template overlaps with a single rectangle from the test pattern. Because there will be no overlap between the remaining rectangles, it will be impossible to identify whether a particular test pattern belongs to the reinforced or nonreinforced category, and subjects should react to all six test patterns in much the same way. For the horizontal group, however, it will be possible to superimpose the two rectangles of a test pattern, albeit only partially, over both rectangles of a template, so that the pattern to which it corresponds can be identified and enable an appropriate response to be made. On completion of this test, a second test was conducted, which was the same as the first except that the gap between the rectangles was increased to twice the length of the long side of the rectangles. With this distance between the rectangles, the best match that both groups will be able to obtain is for one rectangle of the test pattern to overlap with one rectangle of a training pattern, and they will both find it impossible to discriminate between the test patterns.

A different prediction is made if it is assumed the solution of the discrimination depends on the acquisition of a structural description of each training pattern. For both groups, this description should be based on the same relational information (e.g., color A to the left of color B), and performance on the test trials will depend on the extent to which the change to a training pattern affects the ability to appreciate this relationship. Although it is difficult to specify precisely how the changes to the patterns will affect the perception of the spatial relationship between the rectangles, it is reasonable to suppose it will be affected to the same degree in both groups. In other words, if separating the rectangles for the first test should make it difficult for the vertical group to perceive the spatial relationship between the rectangles in a pattern, a similar effect should be found in the horizontal group.

\section{Method}

Subjects. The subjects were 24 experimentally naive adult homing pigeons. The pigeons were housed in pairs and had free access to water and

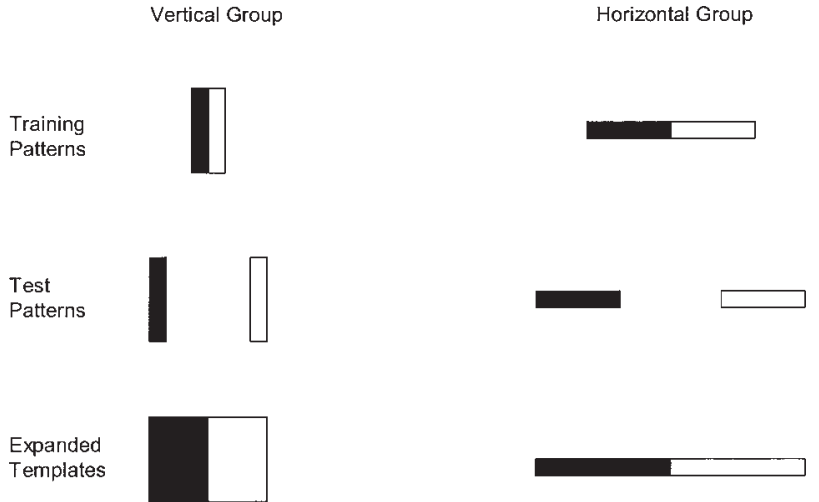

Figure 1. Patterns used for discrimination training with the vertical and horizontal groups of Experiment 1 (top row), the test trials with these groups (middle row), and the expanded templates for the two groups that are considered in the discussion to Experiment 1. The black and white filling of the rectangles represent two of the three colors used in the experiment. 
grit in their home cages. They were maintained at $80 \%$ of their free-feeding weights by being fed a restricted amount of food after each experimental session. They were maintained in a lightproof room in which the lights were on for $14.5 \mathrm{hr}$ each day. At the start of the experiment, they were assigned at random and in equal numbers to the two groups.

Apparatus. The experimental apparatus consisted of eight pigeon test chambers $(30.0 \times 33.0 \times 35.0 \mathrm{~cm})$. Each contained an 8.3-cm-high $\times$ 6.5 -cm-wide clear acrylic panel, which was hinged at the top. Pecks on the panel were detected by a reed relay that was operated whenever a magnet attached to its lower edge was displaced by a distance greater than $1 \mathrm{~mm}$. The midpoint of the panel was $24 \mathrm{~cm}$ above the floor of the chamber. A Sony color TV with a $10 \times 7.5-\mathrm{cm}$ screen was located $2.0 \mathrm{~cm}$ behind the acrylic panel. Food was delivered by operating a grain feeder (Colbourn Instruments, Lehigh Valley, PA) with an opening that measured $5.0 \mathrm{~cm} \times$ $6.0 \mathrm{~cm}$ located in the same wall as the response key. The midpoint of the opening was $9.0 \mathrm{~cm}$ above the chamber floor and $7.0 \mathrm{~cm}$ to the left of the midline of the wall. The feeder was illuminated whenever grain was made available. The chambers were permanently lit during all experimental sessions by a $2.8-\mathrm{W}$ bulb, operated at $24 \mathrm{~V}$, located $2.5 \mathrm{~cm}$ above the top of the acrylic panel. Risc PC microcomputers (Acorn Computers Ltd., Cambridge, England), which were programmed in Arachnid (Paul Fray Ltd., Cambridge, England), were used for the control of events, recording of responses, and generation of stimuli on the TV screens.

Stimuli. The stimuli were presented on the TV screen, which was otherwise dark. The stimuli consisted of rectangles $(24 \mathrm{~mm} \times 3 \mathrm{~mm})$ joined together along a vertical side. The midpoint of the common side of the rectangles was located at the center of the TV screen. For the vertical group, the two rectangles were presented vertically and joined by their long sides; for the horizontal group, the rectangles were presented horizontally and joined by their short sides (see Figure 1). The colors of the rectangles were red, green, and blue. A full list of the training patterns is shown in Table 1.

Procedure. The subjects first received three sessions in which they were trained to eat food whenever it was presented by the hopper. They were then given nine sessions of autoshaping in which a white circle with a diameter of $16 \mathrm{~mm}$ was presented in the middle of the TV screen for $10 \mathrm{~s}$.
There were 45 trials in a session, the mean interval between the start of each trial was $60 \mathrm{~s}$ (range $=35-85 \mathrm{~s}$ ), and food was made available in the hopper whenever the white circle was removed from the TV screen. Whenever food was made available at the hopper, it was presented for $4 \mathrm{~s}$. The remaining sessions of the experiment involved discrimination training with the patterns listed in Table 1. Unless stated otherwise, each of the six patterns was shown eight times in a session. The patterns were presented for $10 \mathrm{~s}$ at a time. For half the subjects in each group, the patterns in the left-hand column of Table 1 were paired with food, and for the remaining subjects those in the right-hand column were paired with food. The patterns were presented in a random sequence, with the constraints that every pattern was shown once in each successive block of six trials and that no more than three reinforced or three nonreinforced trials could occur in succession. The mean interval between the onset of successive patterns was $60 \mathrm{~s}$ (range $=35-85 \mathrm{~s}$ ).

Test trials were conducted in Sessions 20 and 24. In both test sessions, training was conducted as normal for the first 24 trials. The test patterns were then presented randomly intermixed among the training patterns during the remaining 24 trials of the session. The test trials for the first test session for both groups consisted of two presentations of each of the six training patterns but with a gap of $24 \mathrm{~mm}$ between the two rectangles of each pattern. The midpoint of the gap was located at the midpoint of the screen. The test trials were differentially reinforced with food presented after the test patterns based on the training patterns that signaled food but not after the test patterns based on the nonreinforced training patterns. The details of the second test session were the same as for the first, except that the distance between the rectangles of the test patterns for both groups was $48 \mathrm{~mm}$.

All statistical tests were evaluated with respect to an alpha level of .05.

\section{Results}

Figure 2 shows the mean rates of responding per minute for the two groups in the presence of the reinforced and nonreinforced patterns for each of the 24 sessions of training. Responding was

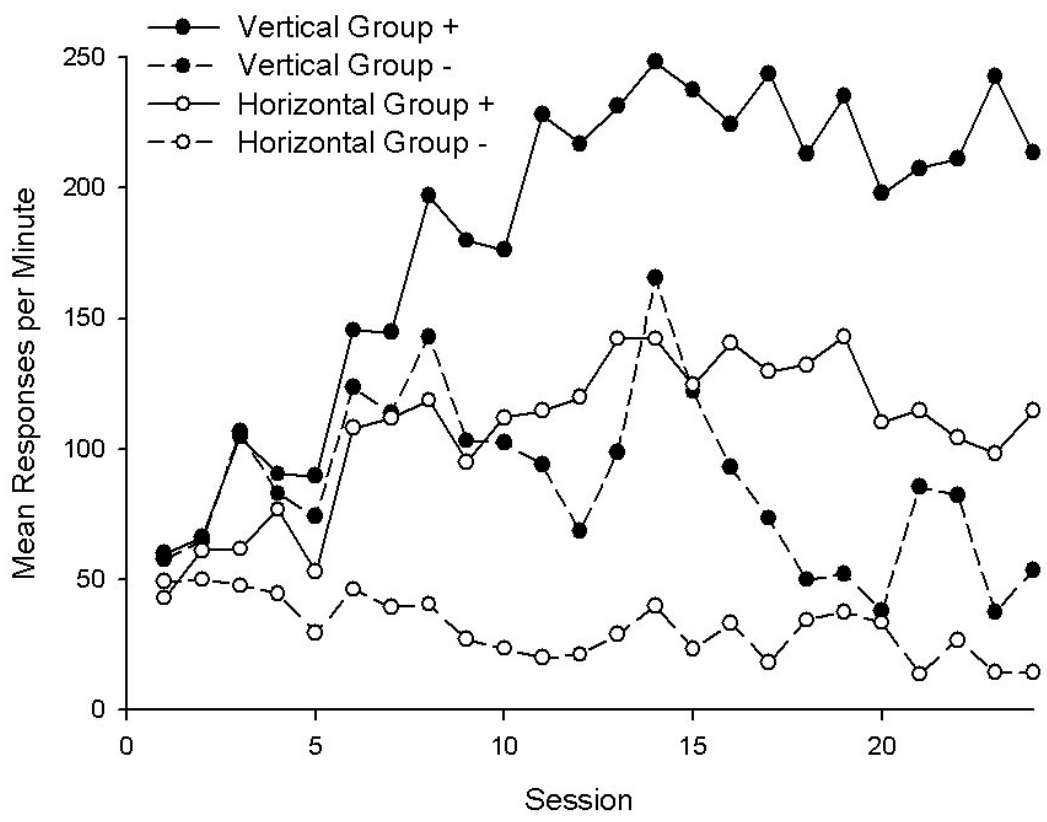

Figure 2. The mean rates of responding by the vertical and horizontal groups of Experiment 1 during the patterns that signaled food $(+)$ or no food $(-)$. 
generally faster during both types of trials by the vertical than the horizontal group. The two groups acquired the structural discrimination, and both discriminated successfully between each of the three pairs of patterns. Thus, during the first test session, Session 20 , the mean number of responses per minute that were recorded by the vertical group during the reinforced and nonreinforced trials were, respectively, 210 and 54 for the blue and green training patterns, 186 and 30 for the green and red training patterns, and 182 and 24 for the red and blue patterns. For the horizontal group, these values were, respectively, 114 and 48 for the blue and green patterns, 102 and 36 for the green and red patterns, and 102 and 12 for the red and blue patterns. Responding during the reinforced patterns by each group was significantly faster than during the nonreinforced patterns for each of the three discriminations, $t \mathrm{~s}(11)>3.42$.

The trials with the different test patterns took place in different sessions. Accordingly, separate analyses were conducted for the two sessions; the rates of responding during the test patterns were compared with those during the training patterns. The mean rates of responding during the reinforced and nonreinforced trials with the training and the test patterns for the first test session are shown in the left-hand panel of Figure 3. The two pairs of histograms in the left half of this panel show the results for the vertical group. There was a clear discrimination between the training patterns that signaled the presence and absence of food (left-hand pair of histograms), but when the same patterns were presented for the test trials with a gap of $24 \mathrm{~mm}$ between the rectangles the discrimination was impaired. The remaining histograms in the left-hand panel of Figure 3 show the results for the horizontal group. The test trials for this group did not affect the discrimination that was evident with the training patterns. A three-way analysis of variance (ANOVA) of individual mean rates of responding during the reinforced and nonreinforced trials with the training and test patterns revealed a significant Group $\times$ Trial (training or test) $\times$ Stimulus (reinforced or nonreinforced) interaction, $F(1,22)=$
15.27. Simple effects analyses revealed a significant Trial $\times$ Stimulus interaction for the vertical, $F(1,22)=38.59$, but not the horizontal $(F<1)$ group, which confirms that the discrimination was disrupted by the change to the patterns for the test trials to a greater extent for the vertical than for the horizontal group. In support of this observation, further tests of simple effects revealed that responding by the vertical group during the reinforced test patterns was significantly slower than during the equivalent training patterns, whereas the opposite outcome was found for the nonreinforced patterns, $F_{\mathrm{s}}(1,44)>13.01$. The equivalent comparisons for the horizontal group were not significant $(F \mathrm{~s}<1.07)$. The simple effects analyses also revealed that for both groups, responding during the test patterns was significantly faster during the reinforced than the nonreinforced stimuli, $F \mathrm{~s}(1,44)>5.86$, which indicates that separating the rectangles by $24 \mathrm{~mm}$ did not abolish the discrimination for either group. The effect of group, stimulus, and the Trial $\times$ Stimulus interaction were all significant, $F \mathrm{~s}(1,22)>11.05$, but the effect of trial and the two remaining interactions were not, $F_{\mathrm{s}}(1,22)<2.36$.

The results from the second test session, which can be seen in the right-hand panel of Figure 3, were much the same as those for the first test, except that the disruption to the discrimination was greater. The three-way ANOVA for the results from the second test session also revealed a significant Group $\times$ Trial $\times$ Stimulus interaction, $F(1,22)=7.33$. In contrast to the previous test, however, a simple effects analysis revealed significant Trial $\times$ Stimulus interactions for both groups, $F_{\mathrm{s}}(1,22)>9.24$, which indicates for each of them that the discrimination was adversely affected by the test trials. The extent of this disruption, however, was greater for the vertical group, which failed to discriminate between the test patterns, $F(1,44)=1.25$, than for the horizontal group, for which the discrimination between the test patterns was statistically significant, $F(1,44)=5.46$. The remaining findings from the three-way ANOVA were significant effects of group, stimulus, and significant Group $\times$ Trial and Trial $\times$ Stimulus

\section{4-mm Test}

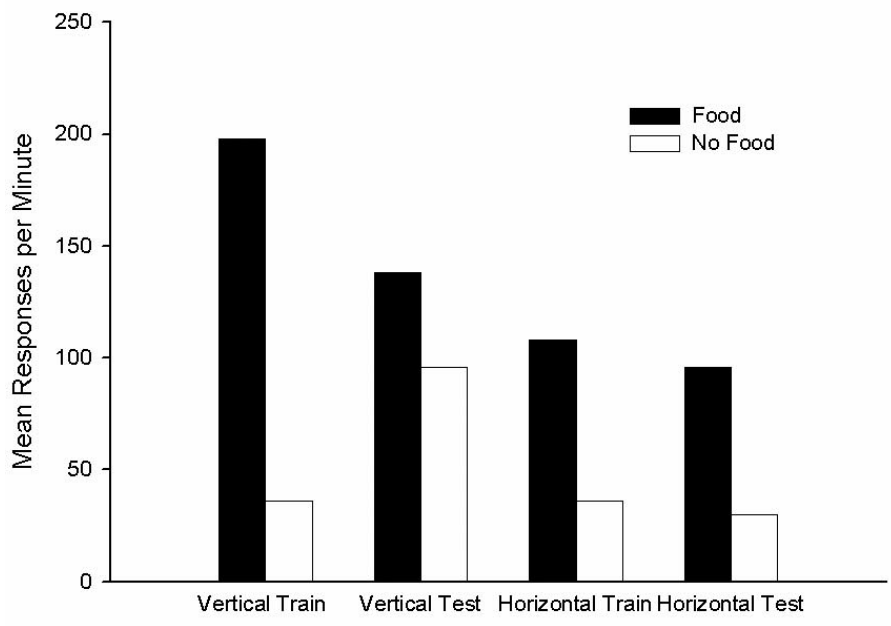

48-mmTest

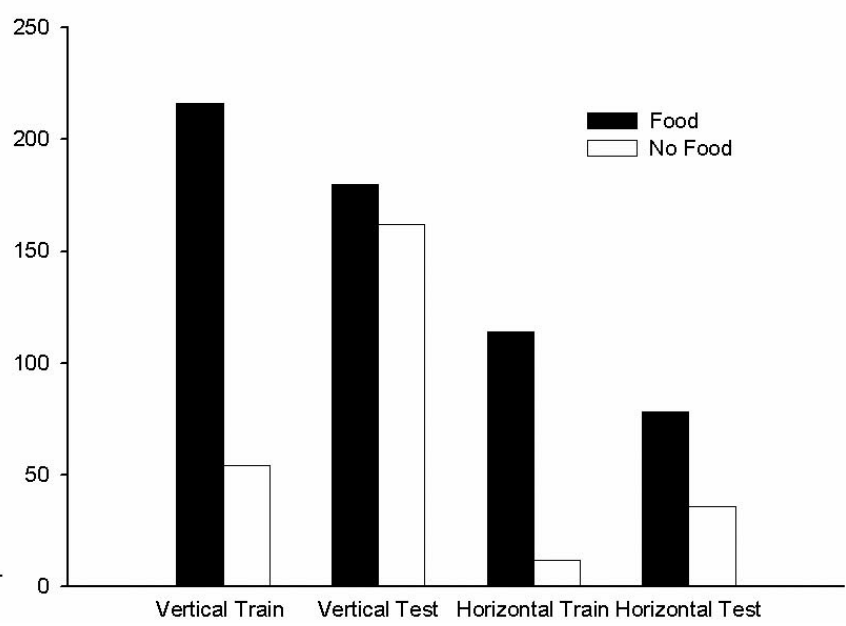

Figure 3. The mean rates of responding during the two test sessions of Experiment 1 by the vertical and horizontal groups in the presence of the training (Train) and test patterns. 
interactions, $F \mathrm{~s}(1,22)>10.45$. The effect of trials and the Group $\times$ Stimulus interaction were not significant, $F$ s $(1,22)<$ 4.10.

\section{Discussion}

We predicted in the introduction that if the solution of the discrimination depends on template matching, then separating the rectangles of the training patterns would affect the discrimination to a greater extent in the vertical than in the horizontal group. Although the results were in keeping with the spirit of this prediction, certain findings from the experiment did not conform to specific predictions that were made. The discrimination between the training patterns was predicted to be abolished by separating the rectangles by $24 \mathrm{~mm}$ for the vertical group and by $48 \mathrm{~mm}$ for the horizontal group. In both cases, these manipulations significantly disrupted the discrimination but did not abolish it.

To deal with this sort of problem, template-matching models of shape and object recognition in humans allow certain transformations of the template so that its match with a test pattern may be improved (Palmer, 1999). Of particular interest to the present results is the suggestion that a template is flexible so that it may be effectively expanded or compressed to provide a better match with a pattern (Tarr \& Bülthoff, 1998). One benefit of being able to make this transformation is that it would enable patterns to be recognized correctly from a variety of viewing distances. It is thus possible that during the test, the templates for the training patterns were expanded until their outer edges coincided with the outer edges of the test pattern. To make the discussion of the present experiments simpler, we assume that this change to the template takes place only in the horizontal plane (which is, of course, the plane in which the training and test patterns differed). This is not to say that the expansion and contraction of a template does not occur simultaneously in both the vertical and horizontal planes or that it may not occur in just the vertical plane. Rather, it is easier to derive precise predictions for the reported experiments by assuming that the change in the template occurs in only the horizontal plane. If the changes to the template are assumed to occur simultaneously in two dimensions, then the predictions that are derived for the following experiments will be altered quantitatively but not qualitatively.

The lower row of Figure 1 shows how the expanded templates for the training patterns for the two groups might be represented. Consider the patterns for the first test session with the vertical group. These patterns comprised two 3-mm wide rectangles separated by a 24-mm gap. To be compared with this pattern, the template of a training pattern would have to be expanded so that it consisted of two colored rectangles that were each $15 \mathrm{~mm}$ wide. If the correct template had been selected, then the colors of the two rectangles of the test pattern would match the colors of the regions of the template that they overlapped, which would allow the test pattern to be identified. The remainder of the template, however, would not match the dark background between the rectangles of the test pattern. To be precise, only $20 \%$ of the correct template would match the test pattern, and thus a considerable generalization decrement would be expected on the test trials of the first test session with the vertical group.

The test patterns for the horizontal group during the first test session were $72 \mathrm{~mm}$ wide and consisted of two 24-mm wide rectangles separated by a 24-mm gap. Once the width of a correct template of a training pattern is increased to $72 \mathrm{~mm}$, its rectangles will match and overlap fully with the two rectangles in the test pattern. Again, the template would not match the background occupying the space between the rectangles, but, on this occasion, the template would correspond with two thirds of the test pattern. The discrimination between the training patterns should, therefore, transfer to the test patterns to a greater extent than for the vertical group. The results confirmed this prediction. A similar prediction is made for the two groups for the second test session, except that the degree of transfer from the training trials to the test trials will be reduced for both of them. This reduction will occur because, compared with the first test, a larger proportion of each test pattern will be composed of the dark background that has no counterpart in the template. To be precise again, $11 \%$ of a test pattern in the vertical group and $50 \%$ of a test pattern in the horizontal group will match the correct templates. The results suggest that the second of these values, but not the first, was sufficient to permit the discrimination to transfer to the test patterns.

The results do not follow so readily from the notion that the solution to this discrimination depends on a structural description being made of each training pattern. If this were true, then separating the rectangles of the training patterns would be expected to have the same effect in both groups. To explain why this was not the case, it would have to be argued that inserting a gap between the rectangles prevented the vertical but not the horizontal group from encoding the spatial relationship between the rectangles. How such an argument could be justified is not clear.

Throughout most of the experiment, the rate of responding during reinforced and nonreinforced trials was faster for the vertical than the horizontal group. This difference between the groups inevitably raises the question of whether it was responsible for the different ways in which the groups reacted on the test trials. For instance, the high rate of responding by the vertical group may have engendered a performance ceiling that made it difficult for responding to be faster during the reinforced than the nonreinforced test patterns. However, the rate of responding by the vertical group during the reinforced test patterns was considerably slower than during the training patterns on which they were based, which should have permitted a discrimination between the test patterns to be detected. In any case, the findings from the next experiment indicate that it would be a mistake to attribute the failure of the vertical group to discriminate between the test patterns to the high rate of responding during the training patterns. This failure is more likely, instead, to be a consequence of the nature of the training patterns.

\section{Experiment 2}

The discriminations in the previous experiment transferred well to the test trials when the boundary between the rectangles of the training patterns was short but transferred poorly when this boundary was long. Although this outcome is consistent with predictions based on the assumption that templates were acquired of each training pattern, it is possible that the length of the boundary between the rectangles was principally responsible for determining performance on the test trials. For instance, when the boundary between the rectangles is long, the edge created by the two colors might be very salient and gain considerable control over the 
discrimination. When this boundary is short, however, the salience of the edge might be low and exert little influence on the discrimination. Removing the boundary, by separating the rectangles of the test patterns, would then result in a greater disruption of performance on the test trials for the group trained with a long boundary. The purpose of Experiment 2 was to determine whether it is worth considering seriously an explanation of this sort for the results of the previous experiment. At the same time, the experiment provides a further opportunity to test the template-matching explanation offered for the findings reported thus far.

There were four groups in the experiment, which received a spatial structural discrimination based on the patterns shown in Figure 4. The discriminations given to the vertical and horizontal groups were the same as for those in Experiment 1. For the remaining two groups, the colors for the structural discrimination were presented in adjacent squares. The lengths of the short and long sides of the rectangles determined the lengths of the sides of the squares for, respectively, the small-square and large-square groups. After discrimination training, the four groups received test trials; the components of their respective training patterns were separated by a distance equal to the length of the long side of the rectangles, or $24 \mathrm{~mm}$.

If the length of the boundary between the two colors of a training pattern is principally responsible for determining the outcome of the test trials, then two different predictions can be made. First, it is possible that the absolute length of this boundary determines the outcome of the test trial, so that the greater the length of this boundary during training the more disruptive will be the effect of removing it on the test trials. If this possibility is correct, then the discrimination between the two sets of patterns by the vertical and the large-square groups should be poor on the test trials, whereas for the horizontal and small-square groups, the discrimination should transfer well to the test patterns. Second, it is possible that the length of the boundary between the colors relative to the length of an adjacent side determines the outcome of the test trials. For instance, when the boundary is longer than the adjacent side, separating the components of the patterns might severely disrupt the discrimination. This manipulation, however, might be less disruptive when these two dimensions are equal and least disruptive when the length of the boundary is less than that of the adjacent side. According to this analysis, therefore, the vertical

Vertical Group

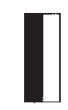

Small-Square Group

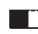

Horizontal Group
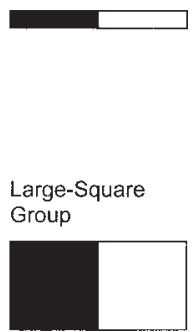

Figure 4. Patterns used for the four groups of Experiment 2. The black and white filling of the rectangles and squares represents two of the three colors used in the experiment. group will show the poorest discrimination between the test patterns, the horizontal group will show the best discrimination, and the results for the small- and large-square groups should be similar and fall between these extremes.

A different outcome to the experiment will be found if the solution of a structural discrimination relies on template matching. In view of the results from the previous experiment, it will be assumed again that a template for a training pattern can be expanded to improve its match with a test pattern. The method of testing for the horizontal and vertical groups was the same as for the previous experiment. According to the analysis offered in the previous discussion, the test patterns of the present study will correspond with $66 \%$ of the expanded template for the correct training pattern in the horizontal group and $20 \%$ in the vertical group. These values are inversely related to the proportion of a test pattern that is filled by the dark background between the two rectangles. The greater this proportion, the weaker is the predicted generalization from the training to the test patterns. It is important to note that this proportion will remain constant no matter how high the rectangles are, which means that, for the present study, the outcome of the test trials will be determined by the width, but not the height, of the rectangles and squares. Therefore, if template matching is responsible for the solution of the original discrimination, then the results from the test trials with the small-square group will be the same as for the vertical group, and those for the large-square group will be the same as for the horizontal group.

\section{Method}

Subjects and apparatus. The subjects were 32 experimentally naive adult homing pigeons from the same stock and maintained in the same way as for Experiment 1. Before the start of the experiment, pigeons were assigned at random and in equal numbers to the four groups. One pigeon in each of the vertical, horizontal, and small-square groups ceased responding before the test session, and they were excluded from the experiment. The apparatus was the same as for Experiment 1.

Procedure. Magazine training and autoshaping with a white circle were the same as for Experiment 1. In each of the next 30 sessions, all subjects received eight trials, with each of the six pairs of colors listed in Table 1 and with patterns based on those shown in Figure 4. For the vertical group, the two colors were presented in two adjacent rectangles that were each $3 \mathrm{~mm}$ wide and $24 \mathrm{~mm}$ high; for the horizontal group, the colors filled two adjacent rectangles that were $3 \mathrm{~mm}$ high and $24 \mathrm{~mm}$ wide; for the small-square group, the colors filled two adjacent squares whose sides were $3 \mathrm{~mm}$ long; and for the large-square group, the colors filled two adjacent squares whose sides were $24 \mathrm{~mm}$ long. The rectangles or squares for all groups made contact with each other along a vertical side.

The training for the first 24 trials of the final test session of the experiment was the same as for the previous sessions. The remaining 24 trials comprised an equal number of trials with the training patterns and the test patterns. The test patterns for each group were created by separating by $24 \mathrm{~mm}$ the rectangles or squares of the six training patterns. Each of these modified training patterns was presented twice during the 12 test trials. Test patterns were followed by the same outcome as the training patterns on which they were based. The remaining procedural details for both stages of the experiment were the same as for Experiment 1.

\section{Results}

The results from the four groups for the 30 sessions of training are shown in Figure 5. The four groups acquired the discrimination, but those trained with the patterns composed of either vertical 


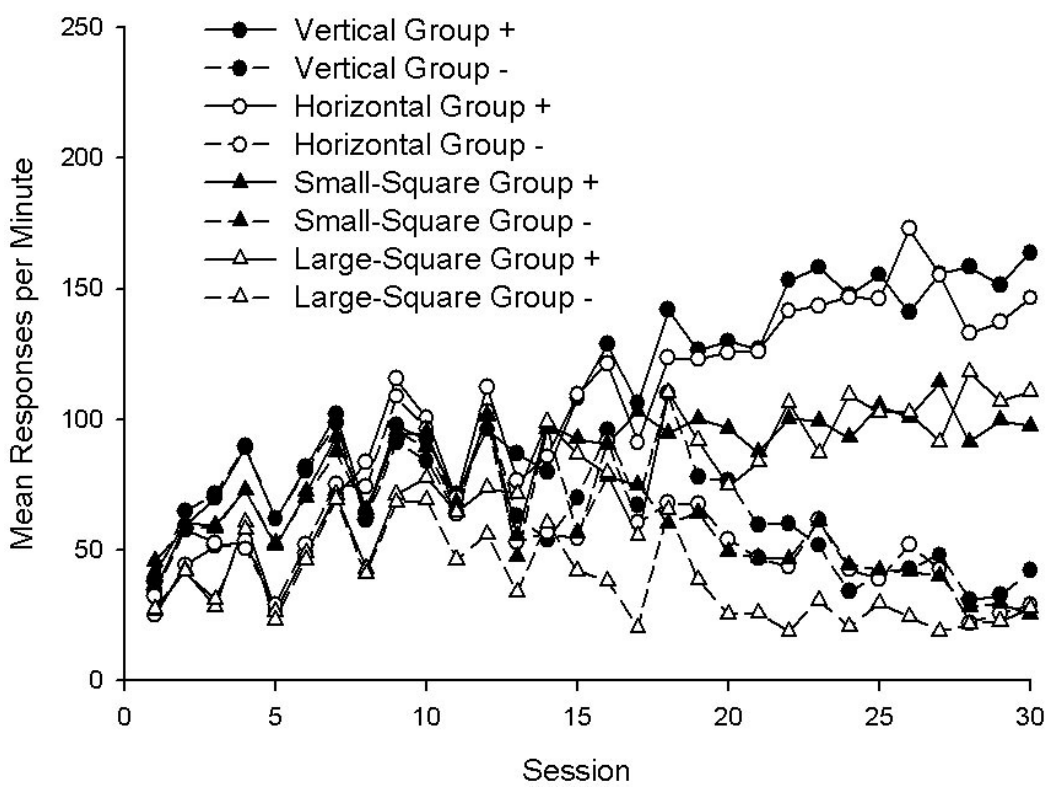

Figure 5. The mean rates of responding by the four groups of Experiment 2 during the patterns that signaled food $(+)$ or no food $(-)$.

or horizontal rectangles responded more vigorously than those trained with patterns containing either large or small squares. The four groups mastered each of the three discriminations. During the final session of the experiment, for the vertical group, the mean number of responses per minute during the reinforced and nonreinforced training patterns, respectively, were 160.6 and 40.1 for the blue-green patterns, 166.7 and 26.8 for the green-red patterns, and 164.2 and 60.3 for the red-blue patterns; for the horizontal group, 149.0 and 26.7, 145.9 and 35.0, and 143.8 and 25.7, respectively; for the small-square group, 106.9 and 21.1, 96.1 and 27.3 , and 88.9 and 28.1, respectively; and for the large-square group, 106.9 and 27.7, 104.8 and 26.6, and 120.8 and 29.0, respectively. For all groups, responding during the reinforced patterns of each discrimination was significantly faster than during the nonreinforced patterns, $t \mathrm{~s}(6$ or 7$)>2.70$.

Figure 6 shows the mean rates of responding during the reinforced and nonreinforced training trials (left-hand panel) and test trials (right-hand panel) for the four groups during the final session of the experiment. Separating the components of the training patterns disrupted the discrimination in every group relative to their performance during the training trials, but the extent of this disruption was greater for the vertical and the small-square groups than for the horizontal and large-square groups. A four-way ANOVA was conducted using the between-group factors of width
Training

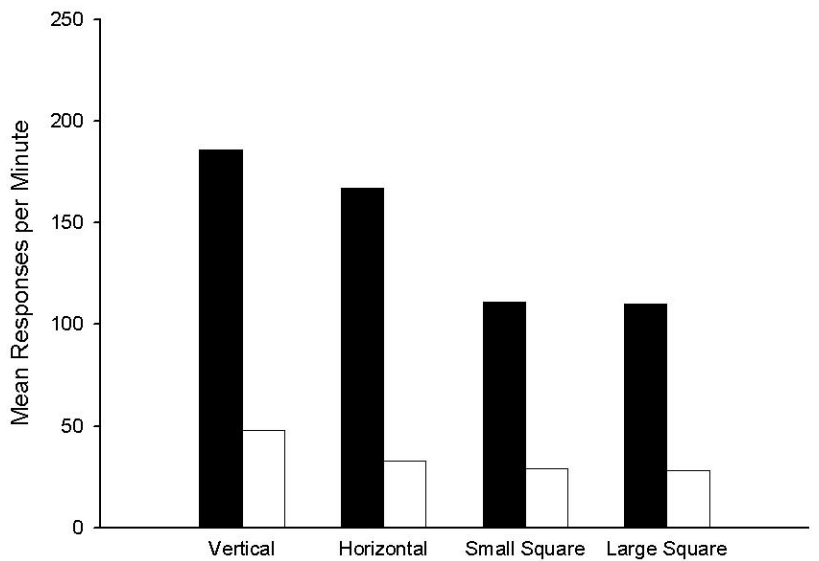

Test

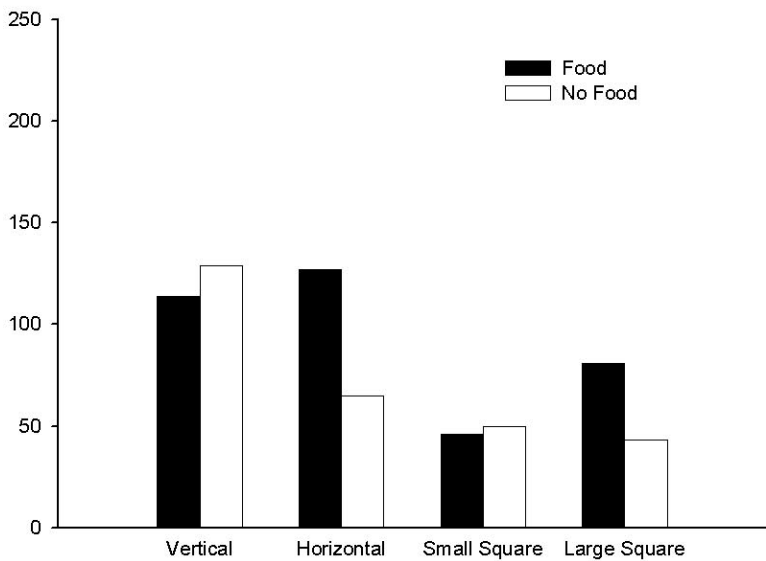

Figure 6. The mean rates of responding by the four groups during the training and test trials in the final session of Experiment 2. 
(whether the components of the patterns were wide or narrow) and shape (whether the components of the patterns were square or rectangular) and the within-group factors of trial (training or test) and stimulus (reinforced or nonreinforced). The four-way interaction was not significant $(F<1)$; the Width $\times$ Trial $\times$ Stimulus interaction was significant, $F(1,25)=5.46$, but the remaining three-way interactions were not significant, $F \mathrm{~s}(1,25)<3.31$. The significant Width $\times$ Trial $\times$ Stimulus interaction indicates that the discrimination was affected more when the components of the patterns were separated by $24 \mathrm{~mm}$ in the groups trained with components that were narrow (vertical rectangles or small squares) rather than wide (horizontal rectangles or large squares). Exploration of the significant interaction with tests of simple effects indicated that there was a significant Width $\times$ Stimulus interaction for the test trials, $F(1,50)=6.31$, but not for the training trials $(F<1)$. During the test trials, the difference between the response rates to the reinforced and nonreinforced patterns was significant for the horizontal and large-square groups combined, $F(1,50)=$ 8.87 , but not for the vertical and the small-square groups combined $(F<1)$

To return to the four-way ANOVA, the Trial $\times$ Conditioned Stimulus interaction was significant, $F(1,25)=46.71$, but the remaining two-way interactions were not $(F \mathrm{~s}<2.27)$. The effects of shape and stimulus were significant, $F_{\mathrm{s}}(1,25)>7.59$, but the effects of trial and width were not $\left(F_{\mathrm{s}}<1\right)$.

\section{Discussion}

Inserting a relatively large gap between the components of the training patterns completely abolished the discrimination for the vertical group and the small-square group, whereas this manipulation had only a modest influence on the discrimination for the horizontal group and the large-square group. This pattern of findings is entirely consistent with predictions drawn earlier from the premise that template matching is responsible for the solution of the discrimination during the training stage. A further implication of the results is that the length of the shared boundary between the components of the training patterns is not directly responsible for determining the degree to which separating these components will disrupt the discrimination. If the length of the shared boundary had been important, then the results from the test trials with the vertical group should have been similar to those for the large-square group. In contrast to this prediction, there was a clear difference between the test results from these groups. It was also suggested that the length of the shared vertical boundary relative to the length of the horizontal side of each component of a pattern might determine the outcome of the test trials. The ratio of these two lengths was the same for the small-square and the large-square groups, yet there was a difference between their performance on the test trials.

In keeping with the results from the previous experiments, the results are again difficult to explain if it is accepted that the solution of the training discrimination depends on each pattern being represented by a structural description. Consider, for example, the results from the small- and large-square groups. The training patterns for these groups differed only in their size, yet in order for an account based on structural descriptions to be correct, it would have to follow that separating the small squares made it more difficult to perceive the spatial relationship between them than when the large squares were separated. There is no obvious reason why the ability to perceive the structural relationship between the components of the test patterns should be affected in this manner.

Figure 5 shows that toward the end of discrimination training, the response rate during the reinforced patterns was greater for some groups than for others. Although this outcome is difficult to explain, it is important that, of the two groups that responded most rapidly during the reinforced training patterns, one group showed a good discrimination between the test patterns, whereas the other group showed a poor discrimination. The same is true for the other two groups that showed a more modest rate of responding during the training patterns. This pattern of results points to the conclusion that the outcome of the test trials with the four groups was not determined by the rates of responding during the training trials. A similar conclusion can be drawn for Experiment 1. In that study, the vertical group responded more rapidly than the horizontal group during the reinforced training patterns, whereas in the present study, the equivalent groups responded at similar rates. Given that the discrimination between the test patterns was better for the horizontal than for the vertical group in both experiments, it seems unlikely that this result in Experiment 1 was a consequence of the different response rates during the training patterns.

Separating the vertical rectangles by $24 \mathrm{~mm}$ disrupted, but did not abolish, the discrimination in Experiment 1, but it completely abolished the discrimination in the present experiment (and, as will be seen, had a similar effect in Experiment 3). We are unable to offer a good explanation for this difference. Nonetheless, in each experiment, the critical issue has been whether the test trials would be more disruptive for the vertical than for the horizontal group. This prediction has been confirmed on both occasions.

The results from both experiments have been explained by assuming that templates were formed of the training patterns. Another explanation for the findings can be developed by referring to the changes that were made to the training patterns in order to create the test patterns. Consider the test trials in which the rectangles of the vertical and horizontal groups were separated by $24 \mathrm{~mm}$. This manipulation for the vertical group would result in each rectangle occupying a new region of the screen that was 9 $\mathrm{mm}$ from its original location, whereas for the horizontal groups, the rectangles of the test patterns would overlap to some extent with regions of the screen previously occupied by the training patterns. Perhaps, therefore, the differences between the results of the test trials for the two groups occurred because of differences in the extent to which the components of the training patterns occupied new regions of the screen. In support of this possibility, Kirkpatrick-Steger, Wasserman, and Biederman (1998, p. 36) cite findings from a pilot study in which the effects of training with a picture displayed in one region of a TV screen transferred poorly when the picture was shown in a new region of the screen. However, results from the final test trial in the next experiment suggest that the effects of training with patterns used for the vertical groups of the first two experiments transfer with only slight disruption when they are shown in a new location. Obviously, it would be more satisfactory if Experiments 1 and 2 had included a test for the effects of moving the patterns to a new position, but the results from Experiment 3 suggest that the disruptive effects of such tests would have been slight and less than the effects of separating the rectangles for the vertical groups. Thus, it seems likely that some factor other than presenting com- 
ponents of the patterns in new locations on the screen was responsible for the disruption of the discrimination that was seen during the test trials in Experiments 1 and 2.

\section{Experiment 3}

The purpose of this final experiment was to test the explanation on the basis of template matching that was offered for the outcome of the test trials from the previous experiments. After subjects had received a spatial structural discrimination with two adjacent vertical rectangles, it was found that separating them by a gap that was equal to their height severely disrupted the discrimination. The explanation offered for this outcome is that the expanded templates for the training patterns would not contain any regions that corresponded with the gap between the rectangles of the test patterns. As a consequence, a point-for-point match between the two patterns would reveal that they were only distantly related, and generalization from a training to a test pattern would be slight. To test this analysis, the vertical group of the present experiment received the same training patterns as those in Experiments 1 and 2 , whereas for the gap group, the rectangles were separated by a gap of $24 \mathrm{~mm}$. On completion of this training, test trials were given in which the distance between the rectangles was increased by 24 $\mathrm{mm}$ for both groups.

The discussion to Experiment 1 has already considered in some detail the effect of separating by $24 \mathrm{~mm}$ the rectangles of the training patterns for the vertical group. This manipulation will result in only $20 \%$ of a test pattern matching the expanded template for the correct training pattern. A different prediction is made for the effect of separating by a further $24 \mathrm{~mm}$ the rectangles of the training patterns for the gap group so that the overall width of each pattern is now $54 \mathrm{~mm}$. We assume that when a template is expanded, the ratio of the width of a rectangle to the overall width of the template remains the same as for the original pattern. Hence, the width of each rectangle in the expanded template will be 5.4 $\mathrm{mm}$, and the gap between them will be $43.2 \mathrm{~mm}$. These values for a test pattern will be, respectively, $3 \mathrm{~mm}$ and $48 \mathrm{~mm}$, so that the correspondence between a correct template and a test pattern will be close but not perfect. In fact, $91 \%$ of test patterns will correspond with the template. The clear prediction from this analysis is that the discrimination between the reinforced and nonreinforced test patterns will be more marked for the gap than the vertical group.

To provide a further test of the rationale behind the predictions that have just been made, the second test involved patterns that, for both groups, consisted of rectangles separated by $12 \mathrm{~mm}$. Thus, the distance between the training patterns was increased by $12 \mathrm{~mm}$ for the vertical group and reduced by $12 \mathrm{~mm}$ for the gap group. Because the extent of the transformation of the training patterns was equivalent for both groups, and because they were tested with the same patterns, it seems natural to anticipate that their performance on the test trials would be affected to the same extent. This prediction does not follow, however, if the solution of a structural discrimination relies on the formation of templates. Using the reasoning presented previously, a template for a correct training pattern will overlap with $33 \%$ of the appropriate test pattern for the vertical group. For the gap group, the templates of the training patterns will have to be contracted in order to ensure that their outer edges coincide with the outer edges of the test patterns. Once

this transformation has taken place, $84 \%$ of a test pattern will overlap with the correct template. Thus, the discrimination between the test patterns is again predicted to be superior for the gap than for the vertical group.

The third test involved trials in which the vertical group was presented with the training patterns for the gap group, and the gap group was presented with the training patterns for the vertical group. On this occasion, the percentage of overlap between the transformed template of a training pattern and a test pattern will be the same for both groups-20\%-and, according to a templatematching analysis, they will perform similarly during the test patterns

The fourth test consisted of trials in which the rectangles for both groups were separated by $6 \mathrm{~mm}$. The degree of match between a transformed training pattern and the test pattern generated from that training pattern is $50 \%$ for the vertical group and $70 \%$ for the gap group. This difference may be insufficient to reveal a difference between the groups, but they both might be expected to show a discrimination between the test patterns and to demonstrate a decrement relative to their performance during the training patterns.

The final test was conducted in order to evaluate an explanation for the results from the first two experiments that was raised in the discussion to Experiment 2. It was suggested that the disruption to the discrimination on the test trials with the vertical groups of those experiments was a consequence of generalization decrement brought about by components of the training patterns appearing in a new region of the screen. To evaluate this explanation, the vertical group received two types of test trials in the final session of the experiment (see the left-hand column of Figure 7). On one type of trial-the transformation test-the left-hand rectangle of the training patterns remained in the same place, and the right-hand

Vertical Group

Training
patterns

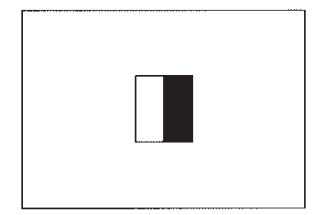

Transformation

test

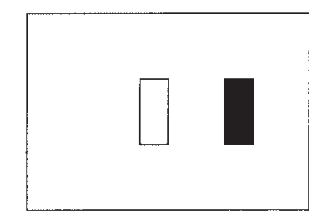

Displacement

test

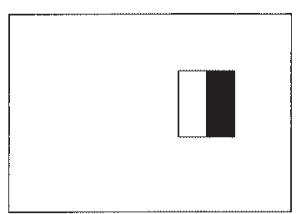

Figure 7. The arrangement of the patterns on the TV screen for the training trials and the two types of test trials in the final session of Experiment 3. The black and white rectangles represent two of the three colors used for the trials. 
rectangle was moved $24 \mathrm{~mm}$ to the right. This manipulation was expected to impair the discrimination. On the other type of trial - the displacement test-both rectangles were moved, as one, to a new location $24 \mathrm{~mm}$ to the right of where they were shown during training. If the impairment seen on the transformation test is a consequence of one of the rectangles appearing in an unfamiliar location, then a similar or even more extreme disruption will be seen on the displacement test because both elements of the test pattern will be presented in new positions. Alternatively, if the extent to which a test pattern matches a template of a training pattern determines the outcome of the test trials, then the transformation test will result in a more severe disruption of the discrimination than the displacement test.

The gap group also received a transformation and displacement test in the final session (see the right-hand column of Figure 7). For the former, the rectangles were presented together, with one of them occupying a new region of the screen. For the latter, they were presented separated by the usual distance of $24 \mathrm{~mm}$ but in a novel position on the screen. The predictions concerning the outcome of these tests are the same as those derived for the vertical group.

\section{Method}

Subjects and apparatus. The subjects were 20 experimentally naive adult homing pigeons that were maintained in the same manner as for Experiment 1. One subject was omitted from the vertical group because it ceased responding before the test sessions. The apparatus was the same as for Experiment 1.

Stimuli. The stimuli for the vertical group were the same as those for the vertical group of Experiment 1. The gap group was trained with the same patterns as the vertical group, except that the rectangles were separated by a distance of $24 \mathrm{~mm}$. The midpoint of the training and test stimuli for both groups was coincident with the midpoint of the TV screen.

Procedure. The procedures for the hopper training and for five sessions of conditioning with a single conditioned stimulus consisting of a white circle with a diameter of $2.5 \mathrm{~cm}$ were the same as for Experiment 1 . During the next 63 sessions, subjects received training with the structural discrimination summarized in Table 1. The five tests took place in Sessions $40,46,53,60$, and 63 . The first 24 trials of a test session were conducted in the same manner as for a training session. The remainder of the session contained 12 training trials randomly intermixed among 12 test trials. The patterns for the test trials of the first four test sessions were based on the six training patterns; each variant of a training pattern was presented twice. The distance between the rectangles of the test patterns for the first test was $24 \mathrm{~mm}$ for the vertical group and $48 \mathrm{~mm}$ for the gap group. The test patterns for both groups in the second test session consisted of the training patterns with the rectangles separated by $12 \mathrm{~mm}$. For the third test session, the distance between the rectangles was $24 \mathrm{~mm}$ for the vertical group and $0 \mathrm{~mm}$ for the gap group. In the fourth test session, the distance between the rectangles was $6 \mathrm{~mm}$ for both groups.

Each of the six training patterns was presented once to each group for the six trials of the transformation test and once again for the six trials of the displacement test. The trials for each type of test were intermixed in a random sequence, with the constraint that no more than two trials of the same test could occur in succession. The transformation trials for the vertical group involved moving the right-hand rectangle of each training pattern $24 \mathrm{~mm}$ to the right, whereas the left-hand rectangle remained in the same place as for the training trials. For the displacement trials, both rectangles were moved $24 \mathrm{~mm}$ to the right. Thus, the gap between the rectangles was $24 \mathrm{~mm}$ for the transformation trials and $0 \mathrm{~mm}$ for the displacement trials. The transformation trials for the gap group involved moving the left-hand rectangle of each training pattern so that it was adjacent to the right-hand rectangle; the left-hand rectangle for the displacement trials was in the same place as for the transformation trials, but the right-hand rectangle was moved $24 \mathrm{~mm}$ to the right. Thus, in contrast to the vertical group, the gap between the rectangles for the gap group was $0 \mathrm{~mm}$ for the transformation trials and $24 \mathrm{~mm}$ for the displacement trials. Procedural details that have been omitted from any stage of the experiment were the same as for Experiment 1.

\section{Results}

The mean rates of responding by the two groups in the presence of the reinforced and nonreinforced patterns for the 63 sessions of training are shown in Figure 8. Both groups eventually responded more rapidly during the reinforced than during the nonreinforced trials, and there was only a modest difference between the performance of the two groups. Both groups had solved each of the three discriminations by the time the test trials were introduced. In Session 40, the first test session, the mean number of responses per minute by the vertical group for the reinforced and nonreinforced trials, respectively, were 141.9 and 27.6 for the red and green patterns, 141.8 and 43.9 for the red and blue patterns, and 124.5 and 40.6 for the blue and green patterns. These values for the gap group were, respectively, 175.1 and 66.4 for the red and green patterns, 183.2 and 52.1 for the red and blue patterns, and 175.7 and 66.5 for the green and blue patterns. Responding was significantly faster during the reinforced compared with the nonreinforced trials of each of the three discriminations for both groups, $t \mathrm{~s}(8)>2.74$, or $t \mathrm{~s}(9)>2.82$.

The results from the training and test trials of the first test session can be seen for both groups in the upper left-hand panel of Figure 9. Increasing the distance between the rectangles by $24 \mathrm{~mm}$ abolished the discrimination for the vertical group, whereas this manipulation had only a slight effect on the performance of the gap group. A three-way ANOVA confirmed these observations by revealing a significant Group $\times$ Trial $\times$ Stimulus interaction, $F(1$, $17)=9.13$. Tests of simple effects revealed a significant Trial $\times$ Stimulus interaction for the vertical group, $F(1,17)=23.54$, but not for the gap group $(F<1)$. Responding was significantly faster during the reinforced compared with the nonreinforced training patterns for both groups, $F_{\mathrm{S}}(1,34)>28.60$, and during the test patterns for the gap group, $F(1,34)=22.14$, but not the vertical group $(F<1)$. The remaining findings from the three-way ANOVA were that the effects of group and trial were not significant $(F \mathrm{~s}<1)$, but the effect of stimulus and all the two-way interactions were significant, $F_{\mathrm{S}}(1,34)>4.93$.

The second test (see the upper-right-hand panel of Figure 9) revealed that moving the rectangles apart by $12 \mathrm{~mm}$ abolished the discrimination for the vertical group, whereas the opposite manipulation of moving them together by this distance had rather little impact on responding by the gap group. In support of these observations, a three-way ANOVA similar to the one just reported revealed a significant three-way interaction, $F(1,17)=4.59$. Subsequent tests of simple effects revealed a significant Trial $\times$ Stimulus interaction for the vertical group, $F(1,17)=12.71$, but not the gap group $(F<1)$. Responding was significantly faster during the reinforced than during the nonreinforced patterns for the training trials in both groups, $F_{\mathrm{s}}(1,34)>12.71$, and for the test trials with the gap group, $F(1,34)=13.84$, but not the vertical group $(F<1)$. The remaining findings from the three-way ANOVA were as follows: The effect of stimulus and the Trial $\times$ 


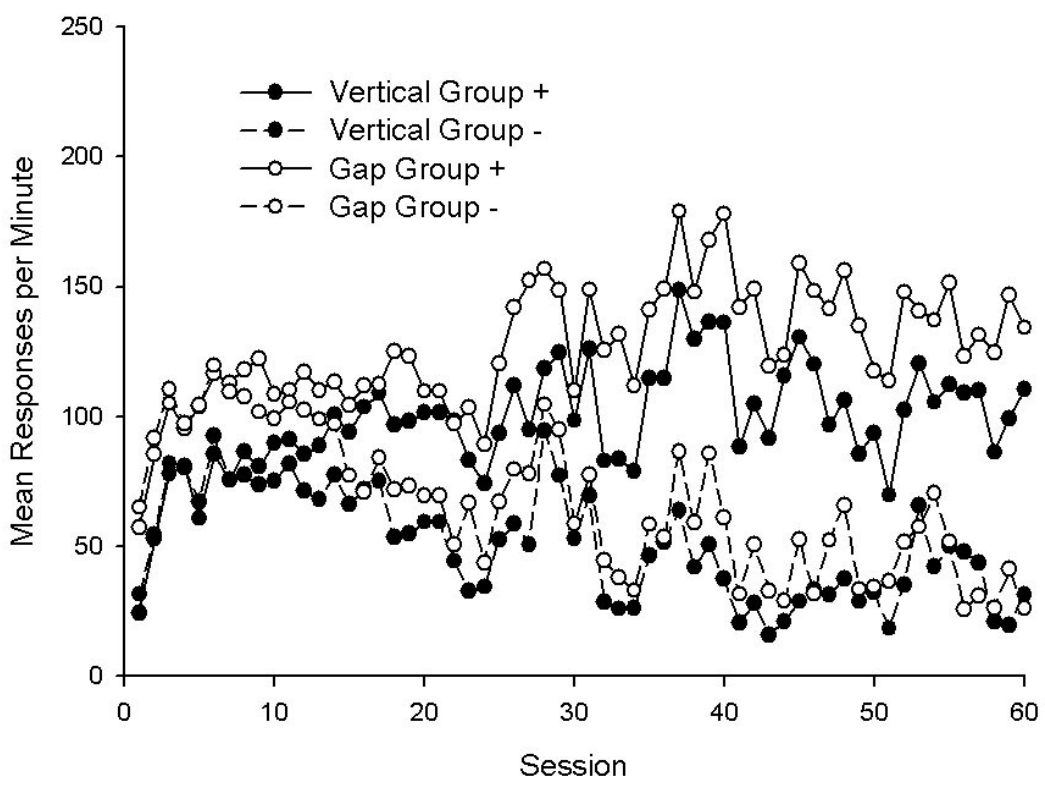

Figure 8. The mean rates of responding by the two groups of Experiment 3 during the patterns that signaled food $(+)$ or no food $(-)$.

Stimulus interaction were significant, $F_{\mathrm{s}}(1,17)>8.41$, whereas the effects of group, trial, and the remaining interactions were not significant, $F_{\mathrm{S}}(1,17)<2.68$.

For the test patterns in Test Session 3, the gap between the rectangles was increased from 0 to $24 \mathrm{~mm}$ for the vertical group and reduced from 24 to $0 \mathrm{~mm}$ for the gap group. (The results from the training and test trials in this session are presented in the lower-lefthand panel of Figure 9.) On the basis of the results presented in Figure 9, it is evident that both manipulations abolished the discrimination between the reinforced and nonreinforced patterns. A three-
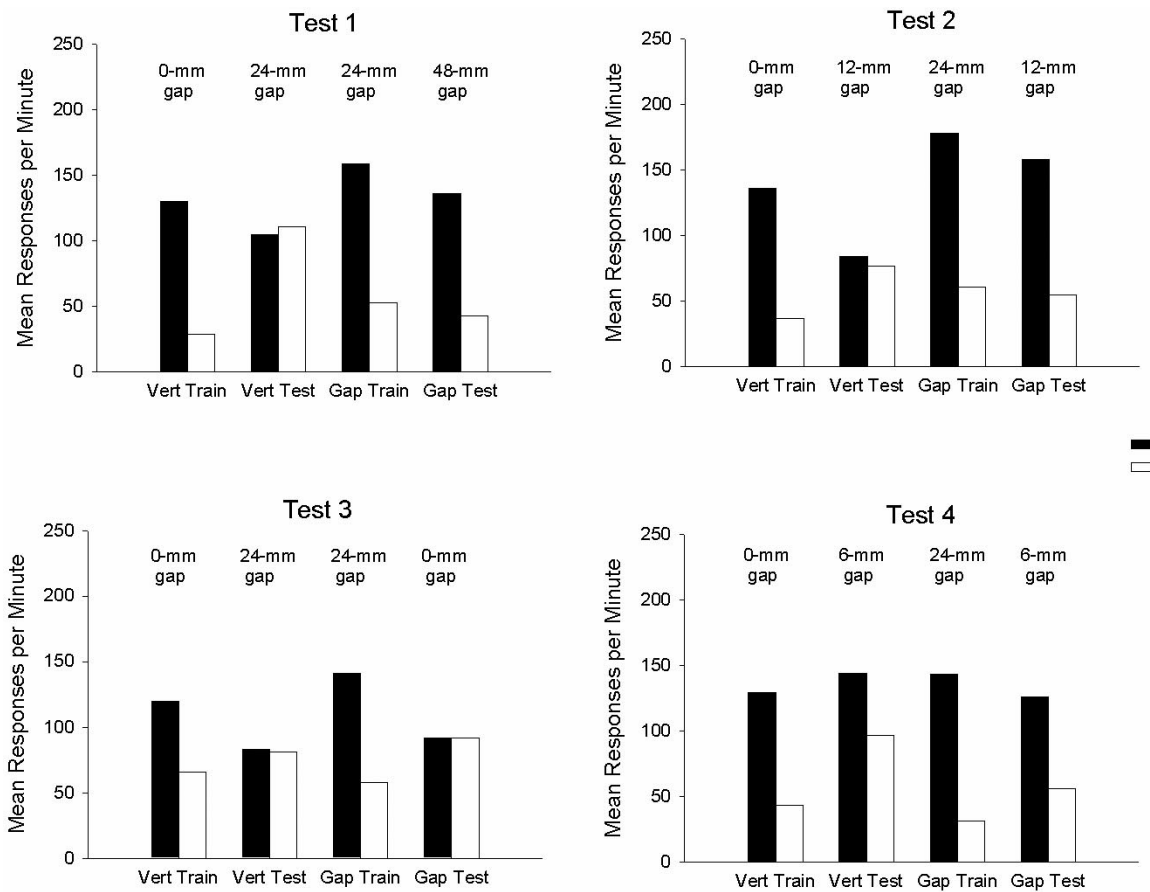

Figure 9. The mean rates of responding during the first four test sessions of Experiment 3 by the vertical (Vert) and gap groups in the presence of the training (Train) and test patterns. The size of the gap between the rectangles for the training and test trials of each test session are shown above the relevant pairs of histograms. 
way ANOVA revealed a significant Trial $\times$ Stimulus interaction, $F(1$, $17)=14.76$. Tests of simple main effects confirmed that, for both groups combined, responding was significantly faster during the reinforced than during the nonreinforced patterns for the training trials, $F(1,34)=33.01$, but not the test trials $(F<1)$. The three-way ANOVA also revealed a significant effect of stimulus, $F(1,17)=$ 19.52 , but the effects of group, test trial, and the remaining interactions were not significant $(F \mathrm{~s}<1)$.

In Test 4 of the experiment, when the rectangles of the test patterns were separated by $6 \mathrm{~mm}$, there was some disruption of the discrimination for both groups (see the lower right-hand panel of Figure 9). In support of this finding, a three-way ANOVA revealed a significant Trial $\times$ Stimulus interaction, $F(1,17)=7.61$. Subsequent tests of simple effects revealed that for both groups combined, responding was significantly more rapid during the reinforced compared with the nonreinforced patterns for the training trials, $F(1,34)=35.00$, and for the test trials, $F(1,28)=14.58$. Further tests revealed that the source of the interaction was a significant increase in the rate of responding from the training to the test trials during the nonreinforced patterns, $F(1,34)=10.92$, but there was no change during these trials with the reinforced patterns $(F<1)$. The ANOVA also revealed a significant effect of trial and stimulus, $F \mathrm{~s}(1,17)>6.69$. The Group $\times$ Trial interaction fell short of the conventional level of significance, $F(1$, $17)=4.42, p=.051$. Subsequent tests of simple main effects revealed that responding on reinforced and nonreinforced trials combined was significantly faster during the test trials than the training trials for the vertical group, $F(1,17)=11.0$, but not for the gap group $(F<1)$. The effect of group and the remaining interactions from the overall ANOVA were not significant $(F \mathrm{~s}<1)$.

Although the three-way interaction fell short of significance, two simple effects analyses based on this interaction were conducted. One analysis was conducted in order to determine whether moving the rectangles closer together for the test trials with the gap group significantly impaired the discrimination. On this occasion, responding during the reinforced test patterns was significantly faster than during the nonreinforced patterns for this group, $F(1$, $34)=4.77$. The other analysis was conducted in order to test for the vertical group whether the discrimination between the test patterns was significantly impaired relative to that recorded during the training patterns. On this occasion, the necessary two-way interaction fell just short of the conventional level of significance, $F(1,17)=4.22, p=.056$.

The results from the final test for the vertical group can be seen in the left-hand panel of Figure 10, and those for the gap group can be seen in the right-hand panel of Figure 10. The discrimination between the reinforced and nonreinforced patterns for both groups was abolished by the transformation test and disrupted to a slight extent by the displacement test. A three-way ANOVA of individual mean response rates for the training trials and the two types of test trial revealed a significant Trial $\times$ Stimulus interaction, $F(2$, $34)=11.60$. Subsequent tests of simple main effects revealed a significant difference between the rates of responding for both groups combined during the reinforced and nonreinforced patterns for the training trials, $F(1,51)=32.61$, and the displacement test, $F(1,51)=17.81$, but not for the transformation test $(F<1)$. The remaining findings from the ANOVA were a significant effect of trial, $F(2,34)=9.24$, and of stimulus, $F(1,17)=22.21$; but the effect of group $(F<1)$, the Group $\times$ Trial interaction $(F<1)$, the Group $\times$ Stimulus interaction, $F(1,17)=1.50$, and the three-way interaction $(F<1)$ were not significant.

To assess whether the effect of the displacement test resulted in a significant impairment of the discrimination relative to that seen during the training trials, a further ANOVA was conducted that was similar to the one just described, except that the results from the transformation test were omitted. The analysis failed to reveal any significant effect of the displacement test. There was a significant effect of stimulus, $F(1,17)=21.24$, but the effect of trial and all the interactions involving trial were not significant, $F_{\mathrm{s}}(1,17)<$ 2.95. The Group $\times$ Stimulus interaction was also not significant, $F(1,17)=1.28$.

\section{Discussion}

Overall, the results from individual test sessions conform well to predictions that were derived from the assumption that the solution of a spatial structural discrimination depends on template match-
Vertical Group

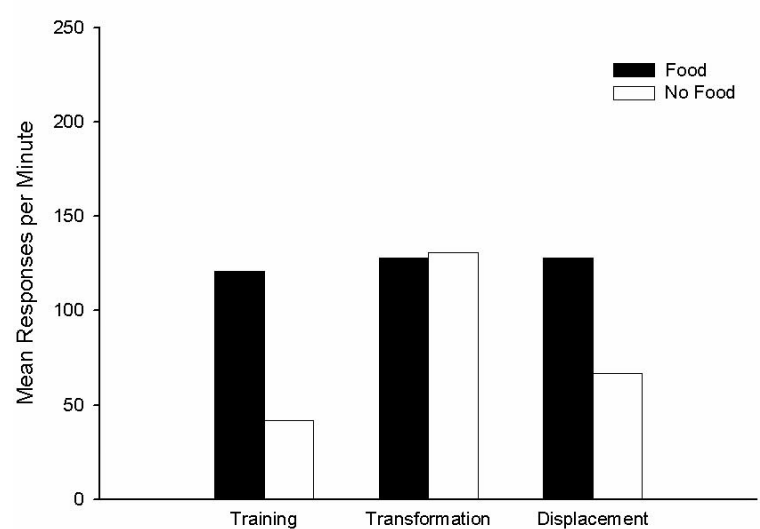

Gap Group

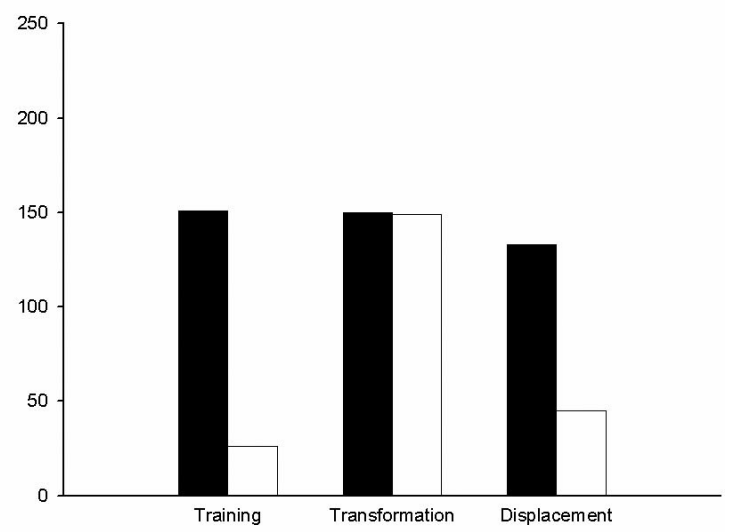

Figure 10. The mean rates of responding by the two groups during the training patterns and the patterns used for the displacement and transformation tests in the final test session of Experiment 3. 
ing. For each of the first four tests, a measure of the degree of overlap between a transformed training pattern and the test pattern derived from it was calculated. If there is any merit in the suggestion that template matching underlies the solution of a structural discrimination, then the value of this measure should be directly related to the magnitude of the discrimination observed with the test patterns. To test this prediction, discrimination ratios were calculated for all subjects for all the test sessions except the one involving the displacement test. The ratio was of the form $\mathrm{A} /(\mathrm{A}+$ $\mathrm{B})$, where $\mathrm{A}$ is the mean rate of responding during the reinforced test patterns, and $\mathrm{B}$ is the mean rate during the nonreinforced test patterns. A group mean was then computed from these values for each of the four test sessions. For the two test sessions with the vertical group in which there was a 24-mm gap between the rectangles, a mean was calculated using the results from both tests. There were, therefore, four group means for the gap group and three group means for the vertical group. These means are displayed in the scatter plot in Figure 11; the horizontal axis represents the percentage of a test pattern that matched the transformed template of the training pattern from which the test pattern was derived. Figure 11 shows a remarkably strong positive correlation between the magnitude of a discrimination ratio and the percentage of match calculated for the test on which the ratio was obtained. It must be acknowledged that the test trials were conducted at different times and not in a counterbalanced sequence. Even so, the orderly relationship exhibited in Figure 11 lends reassuring support to the view that performance on the test trials was indeed controlled by the degree of match between a transformed template and a test pattern. In support of this conclusion, the correlation between the two variables displayed in Figure 11 was significant (Spearman $r=.93$ ).

The results from the experiment do not follow readily from the alternative suggestion that the training patterns were represented as structural descriptions. For example, this account would need to explain why inserting a gap of $12 \mathrm{~mm}$ but not $6 \mathrm{~mm}$ between the rectangles for the vertical group abolished the discrimination for

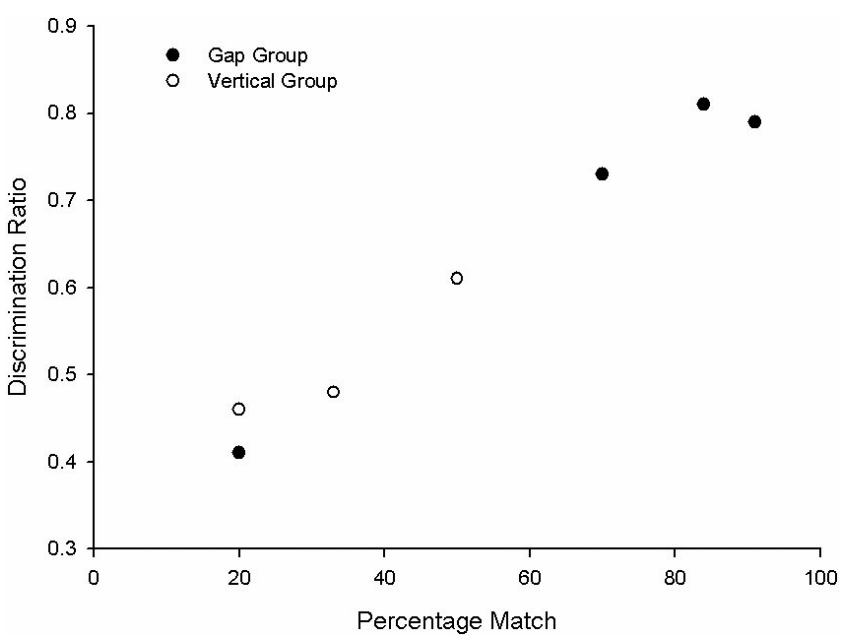

Figure 11. Scatter plot showing the relationship between the group mean discrimination ratios and the percentages of match between the test patterns and the transformed templates of the training patterns for Experiment 3. the vertical group or why the discrimination in the gap group was disrupted to a greater extent by reducing the distance between the rectangles to $6 \mathrm{~mm}$ rather than increasing it to $48 \mathrm{~mm}$. Neither of these outcomes would seem to follow readily if structural descriptions are responsible for pattern recognition. The relationship shown in Figure 11 would also seem to be difficult to explain if structural descriptions are responsible for pattern recognition in pigeons.

The discussion to Experiment 2 raised the possibility that performance on the test trials of the first two experiments was related to whether components of the patterns appeared for the first time in regions of the screen they did not occupy during training. The results from the final test lend no support to this suggestion. Both groups received a displacement test in which the training patterns occupied new regions of the screen without any change to the patterns themselves. These tests revealed for both groups that the discrimination between the two sets of patterns was not significantly affected by this manipulation. In contrast, when the patterns were transformed by moving one of their rectangles to a new region of the screen, then the discrimination between the two sets of patterns was abolished. Thus, a change to the way in which a pattern is constructed, rather than where it is located on the screen, appears to be the more important determinant of how subjects will react on a test trial. Of course, this conclusion is entirely consistent with the proposal that the similarity between a test pattern and the transformed template for a training pattern determines the response to the test pattern.

Some of the results from Experiment 3 can be explained if it is assumed that performance on the test trials is determined by whether or not the rectangles remain together or apart for both the training and the test trials. Consider the gap group, which was trained with the rectangles separated from each other. The discrimination transferred with little disruption to the test patterns of the first two test sessions, for which the rectangles were again separated, but not to the patterns with adjacent rectangles that were used for the third test session. Likewise, for the vertical group, which was trained with adjacent rectangles, the discrimination was completely abolished by separating the rectangles by $12 \mathrm{~mm}$ or more for the first three test sessions. The results from the fourth test session, in which the rectangles were separated by $6 \mathrm{~mm}$ for both groups, however, do not fit so comfortably with the foregoing analysis. This test revealed that separating the rectangles for the vertical group did not abolish the discrimination. It also revealed that, even though the rectangles remained separated for the test with the gap group, reducing the distance between them impaired the discrimination (although this effect fell just short of the conventional level of significance). The results from Experiments 1 and 2 are also difficult to explain if it is accepted that the outcome of the test trials is determined by whether a gap between the rectangles is introduced, or removed, for the test patterns. For instance, the vertical and horizontal groups in both experiments were trained without a gap between the rectangles and tested with a gap. Both groups would, therefore, be expected to fail to discriminate between the test patterns, yet it was found that the horizontal groups showed a clear discrimination between the two sets of test patterns, and that this discrimination was superior to that seen with the vertical group. 


\section{General Discussion}

Pigeons were required in three experiments to discriminate between two sets of patterns on the basis of the spatial relationship between their components. They then received test trials in which the components were moved further apart or closer together. These tests were intended to evaluate the proposal that templates were formed of each training pattern, which could then be expanded or contracted in order to facilitate the classification of the test patterns. In support of this proposal, the degree of overlap between a test pattern and the corresponding transformed template was found to be related to the accuracy with which it could be classified as a signal for reward or nonreward.

Blough (1985; see also Heinemann \& Chase, 1990) has also proposed that discriminations in pigeons are based on templates of the training patterns. However, successful generalization to new patterns was explained by assuming that the template of a training pattern has blurred or fuzzy boundaries. One effect of this blurring is to enable correct classification of patterns that are larger or smaller than the training patterns (Heineman \& Chase, 1990). In Experiment 1, therefore, the discrimination between the rectangles of the vertical group when they were separated by $24 \mathrm{~mm}$ might have occurred because they overlapped with a fuzzy template of the training patterns, even though the test patterns would not overlap with a literal copy of these patterns. By appealing to this type of explanation, it is possible to account for most of the results from the first two experiments, but it is not clear, at least at first glance, how the results from the first test in Experiment 3 could be explained in this way. The test patterns for both groups were created by adding $24 \mathrm{~mm}$ to the distance between the rectangles of the training patterns. If the templates of both sets of training patterns are blurred to the same extent, then the performance by the two groups should have been the same for the test trials. Contrary to this prediction, the discrimination transferred without disruption to the test patterns for the gap group, whereas it was completely abolished on the test trials with the vertical group.

The proposal that a spatial structural discrimination depends on templates for its solution raises the question as to whether all aspects of spatial behavior in animals depend on this means of representing spatial information. There is certainly evidence that template matching plays a role in guiding some animals back to where they have previously found food. Cartwright and Collett (1983) demonstrated with honeybees that changing the size of a landmark near the food source altered in a systematic fashion the distance from the landmark where the bees would search. They, therefore, proposed that honeybees at a food source remember the image created by a view of the surrounding landmarks. Return visits are made possible by moving in a manner that reduces the discrepancy between the current retinal image of a landmark and the remembered snapshot or template. There is also some evidence to suggest that a similar process assists birds to return to a place where they have cached food. Bossema (1979) found that European jays tended to approach food they had hidden from the same direction as when they originally hid it. Such a tendency would be expected if they were attempting to match their current view with the view they experienced during their previous approach to the site. Kamil, Balda, and Good (1999) have reported a similar effect with Clark's nutcrackers. However, these birds were often successful at finding food, even when they approached it from an entirely new direction. Thus, although template matching may play a role in food recovery by birds, Kamil et al. (1999) argue that an additional process is responsible for guiding them to places where they have stored food. They tentatively suggest that this additional process is based on a cognitive map (e.g., O'Keefe \& Nadel, 1978).

The results from rather different studies have also been taken as evidence that templates are not responsible for all aspects of spatial behavior in birds. Cheng (1988) trained pigeons to find food that was hidden in the floor of a square arena at a fixed distance and direction from a blue rectangle attached to one of the walls. Tests in which the width of the rectangle was doubled, or halved, did not affect where subjects searched for food. He argued that these results, which stand in contrast to the findings by Cartwright and Collett (1983), imply that the position of food was identified by a landmark-to-goal vector, which contains information about the direction and distance of the food from the landmark (see also Cheng, 1989). In a related series of experiments, Spetch, Cheng, and MacDonald (1996) required pigeons to peck a touch screen in a small region that was surrounded by four landmarks presented on the screen. When test trials were conducted with an expanded array of landmarks, the birds no longer searched at the center of the array, but they tended to search in a location that was at the original distance and direction of the target region from one of the landmarks. The authors argue that if the birds had identified the location of the target region by means of a template based on the four landmarks, then they should have continued to peck in the center of the array on the test trial. Turning now to the ability of pigeons to find their way home over considerable distances, Biro, Meade, and Guilford (2004) repeatedly released birds from the same site some $10 \mathrm{~km}$ from their loft. They found that the birds returned home by highly stereotyped, relatively inefficient routes. Moreover, when they were released from a novel site that was 1 $\mathrm{km}$ from the route, they would head for the route and then follow it to the loft. Biro et al. (2004) explained these findings by proposing that the pigeons had memorized the images created by a sequence of visual landmarks along the route. It is conceivable that the birds made their way home by using these images to move from one landmark to the next in a similar manner to that proposed by Cartwright and Collett (1983).

The results from these experiments suggest that birds are able to represent spatial information in at least two ways. One is based on templates, which contain implicit spatial information by virtue of being equivalent to a two-dimensional copy of an object or scene. The other involves a more explicit statement of the spatial relationship between two or more objects and may be based on landmark-to-goal vectors (e.g., Cheng, 1989) or a cognitive map (e.g., O'Keefe \& Nadel, 1978). A study in our laboratory, using pigeons with hippocampal lesions, lends support to this claim (Pearce, George, Haselgrove, Erichsen, \& Good, 2005). The birds were not impaired in their ability to solve the spatial structural discrimination that was given to the gap group in Experiment 3, but they experienced considerable difficulty in finding food in an open-field foraging task in which its location could be identified by referring to distant landmarks. One interpretation of these findings is that the hippocampus is not important for using templates to recognize patterns and objects such as landmarks, but it is important for identifying the spatial relationships between such objects as landmarks and goals. Whether this function involves 
heading vectors, cognitive maps, or some other spatial representation remains to be determined. For the present, the findings endorse the conclusion that pigeons have at least two methods for representing spatial information.

The possibility was raised in the introduction of the present article that pigeons use structural descriptions to represent patterns. One problem with this explanation is the lack of evidence showing that pigeons are able to represent spatial information of the sort "A is to the left of B." However, even if it is accepted that they can use such information, nothing is known about the conditions that determine whether or not a particular spatial relationship will be detected. Accordingly, it has not been possible to draw precise predictions from this type of account concerning the present experiments. Our results may, therefore, pose a challenge to the idea that the patterns were identified by means of structural descriptions, but they do not contradict it.

A number of other studies have attempted to determine whether pigeons use structural descriptions to identify complex patterns. In each of these studies, the patterns consisted of line drawings of man-made objects. Wasserman et al. (1993) trained pigeons to discriminate between drawings of, for example, a watering can and a desk lamp. The discrimination between these objects was severely disrupted on test trials in which all the components of an object were presented but rearranged both horizontally and vertically. According to Wasserman et al. (1993), this finding implies that pigeons form structural descriptions of patterns, because such descriptions would allow them to detect that the components of a pattern had been rearranged. Of course, the results could also be explained if the discrimination was solved by forming a template of each training pattern.

A similar ambiguity relates to the interpretation of findings from an experiment by Wasserman et al. (1996). Once pigeons had been trained to discriminate between line drawings of different objects, they were presented with drawings of the same objects but rotated in depth. The discrimination transferred to the new views of the objects, but the extent of this generalization was directly related to the degree of rotation. Furthermore, the slope of the generalization gradient became more shallow if training involved more than one view of the object. The authors acknowledge that these findings are consistent with the claim that pigeons represent patterns and objects as structural descriptions or as templates. According to the former, the amount of generalization is determined by the extent to which a new view introduces new components of the object and new structural relations. According to the latter, the amount of generalization decrement will be related to the degree to which the template of the training pattern matches the test pattern. This matching process is likely to involve some manipulation of the template (Tarr \& Bülthoff, 1998) and will benefit from experience of more than one view of the object (Ullman, 1998).

The final study to consider is by Van Hamme et al. (1992), who trained pigeons to discriminate between line drawings of objects with half of the contour lines deleted. When the birds were tested with drawings of the same objects, but with the original lines deleted and the missing lines restored, they continued to discriminate successfully between them. This result is difficult to explain with a simple account of template matching, in which the similarity between a template and a test pattern is judged on a point-bypoint comparison. However, Van Hamme et al. (1992) show how the more complex account of template matching proposed by
Heinemann and Chase (1990) is able to explain their findings. The authors also show that their findings can be readily explained by Biederman's (1987) recognition by components theory, which is based on structural descriptions of patterns.

The question as to whether pattern recognition in animals depends on templates or structural descriptions thus remains unresolved. In the present article, we have shown that it is possible to derive precise predictions from the assumption that the training patterns were identified by means of templates and to confirm these predictions. For these reasons alone, it seems worth pursuing further the role of templates in both discrimination and spatial learning in animals.

\section{References}

Biederman, I. (1987). Recognition-by-components: A theory of human image understanding. Psychological Review, 94, 115-147.

Biro, D., Meade, J., \& Guilford, T. (2004). Familiar route loyalty implies visual pilotage in the homing pigeon. Proceedings of the National Academy of Sciences, USA, 101, 17440-17443.

Blough, D. S. (1985). Discrimination of letters and random dot patterns by pigeons and humans. Journal of Experimental Psychology: Animal Behavior Processes, 11, 261-280.

Bossema, I. (1979). Jays and oaks: An eco-ethological study of a symbiosis. Behaviour, 70, 1-117.

Cartwright, B. A., \& Collett, T. S. (1983). Landmark learning in bees: Experiments and models. Journal of Comparative Physiology (A), 151, 521-543.

Cheng, K. (1988). Some psychophysics of the pigeon's use of landmarks Journal of Comparative Physiology (A), 162, 815-826.

Cheng, K. (1989). The vector sum model of pigeon landmark use. Journal of Experimental Psychology: Animal Behavior Processes, 15, 366-375.

George, D. N., Ward-Robinson, J., \& Pearce, J. M. (2001). Discrimination of structure: I. Implications for connectionist theories of discrimination learning. Journal of Experimental Psychology: Animal Behavior Processes, 27, 206-219.

Gochin, P. M. (1996). The representation of shape in the temporal lobe. Behavioural Brain Research, 76, 96-116.

Heinemann, E., \& Chase, S. (1990). A quantitative model for pattern recognition. In M. L. Commons \& R. J. Herrnstein (Eds.), Computational and clinical approaches to pattern recognition and concept formation (Vol. 9, pp. 109-126). Hillsdale, NJ: Erlbaum.

Hummel, J. E., \& Biederman, A. (1992). Dynamic binding in a neural network for shape recognition. Psychological Review, 99, 480-517.

Kamil, A., Balda, R. P., \& Good, S. (1999). Patterns of movement and orientation during caching and recovery by Clarks's nutcrackers, Nucifraga columbiana. Animal Behaviour, 57, 1327-1335.

Kirkpatrick-Steger, K., \& Wasserman, E. A. (1996). The what and where of pigeon's processing of complex visual stimuli. Journal of Experimental Psychology: Animal Behavior Processes, 22, 60-67.

Kirkpatrick-Steger, K., Wasserman, E. A., \& Biederman, I. (1998). Effects of geon deletion, scrambling, and movement on picture recognition in pigeons. Journal of Experimental Psychology: Animal Behavior Processes, 24, 34-46.

O'Keefe, J., \& Nadel, L. (1978). The hippocampus as a cognitive map. Oxford, England: Oxford University Press.

Palmer, S. E. (1999). Vision science: Photons to phenomenology. Cambridge, MA: MIT Press.

Pearce, J. M., George, D. N., Haselgrove, M., Erichsen, J. T., \& Good, M. A. (2005). The influence of hippocampal lesions on the discrimination of structure and on spatial memory in pigeons. Behavioral Neuroscience.

Spetch, M. L., Cheng, K., \& MacDonald, S. E. (1996). Learning the configuration of a landmark array: I. Touch-screen studies with pigeons and humans. Journal of Comparative Psychology, 110, 55-68. 
Srinivasan, M. V. (1992). Distance perception in insects. Current Directions in Psychological Science, 1, 22-26.

Tarr, M. J., \& Bülthoff, H. H. (1998). Image-based object recognition in man, monkey, and machine. Cognition, 67, 1-20.

Ullman, S. (1998). Three-dimensional object recognition based on the combination of views. Cognition, 67, 21-44.

Van Hamme, L. J., Wasserman, E. A., \& Biederman, I. (1992). Discrimination of contour-deleted images by pigeons. Journal of Experimental Psychology: Animal Behavior Processes, 18, 387-399.

Wasserman, E. A., Gagliardi, J. L., Cook, B. R., Kirkpatrick-Steger, K., Astley, S. L., \& Biederman, I. (1996). The pigeon's recognition of drawings of depth-rotated stimuli. Journal of Experimental Psychology: Animal Behavior Processes, 22, 205-221.
Wasserman, E. A., Kirkpatrick-Steger, K., Van Hamme, L. J., \& Biederman, I. (1993). Pigeons are sensitive to the spatial organization of complex visual stimuli. Psychological Science, 4, 336-341.

Wehner, R. (1997). Insect navigation: Low-level solutions to high-level tasks. In M. V. Srinivasan \& S. Venkatesh (Eds.), From living eyes to seeing machines (pp. 158-173). Oxford, England: Oxford University Press.

Received November 9, 2004

Revision received April 15, 2005

Accepted April 19, 2005

\section{New Editors Appointed, 2007-2012}

The Publications and Communications (P\&C) Board of the American Psychological Association announces the appointment of three new editors for 6-year terms beginning in 2007. As of January 1, 2006, manuscripts should be directed as follows:

- Journal of Experimental Psychology: Learning, Memory, and Cognition (www.apa.org/journals/ xlm.html), Randi C. Martin, PhD, Department of Psychology, MS-25, Rice University, P.O. Box 1892, Houston, TX 77251.

- Professional Psychology: Research and Practice (www.apa.org/journals/pro.html), Michael C. Roberts, PhD, 2009 Dole Human Development Center, Clinical Child Psychology Program, Department of Applied Behavioral Science, Department of Psychology, 1000 Sunnyside Avenue, The University of Kansas, Lawrence, KS 66045.

- Psychology, Public Policy, and Law (www.apa.org/journals/law.html), Steven Penrod, PhD, John Jay College of Criminal Justice, 445 West 59th Street N2131, New York, NY 10019-1199.

Electronic manuscript submission. As of January 1, 2006, manuscripts should be submitted electronically through the journal's Manuscript Submission Portal (see the Web site listed above with each journal title).

Manuscript submission patterns make the precise date of completion of the 2006 volumes uncertain. Current editors, Michael E. J. Masson, PhD, Mary Beth Kenkel, PhD, and Jane GoodmanDelahunty, $\mathrm{PhD}, \mathrm{JD}$, respectively, will receive and consider manuscripts through December 31, 2005. Should 2006 volumes be completed before that date, manuscripts will be redirected to the new editors for consideration in 2007 volumes.

In addition, the P\&C Board announces the appointment of Thomas E. Joiner, PhD (Department of Psychology, Florida State University, One University Way, Tallahassee, FL 32306-1270), as editor of the Clinician's Research Digest newsletter for 2007-2012. 